\title{
MATHEMATICAL MODELING OF LEACHING PROCESS OF RED MUD IN ORDER TO OBTAIN THE KINETICS PARAMETERS
}

\author{
E. M. Magalhães, \\ K. L. M. dos Passos, \\ B. M. Viegas, \\ E. N. Macêdo, \\ and J. A. S. Souza \\ Universidade Federal do Pará \\ Faculdade de Engenharia Química \\ Bairro Guamá CP. 19011, Belém, Pará, Brasil \\ magalhaes@ufpa.br \\ Received: September 08, 2015 \\ Revised: October 08, 2015 \\ Accepted: November 09, 2015
}

ABSTRACT

The use of the waste of the Bayer process, red mud, is due to its chemical and mineralogical composition that shows a material rich in oxides of iron, titanium and aluminum. Some studies conducted show that this waste can be applied as a source of alternative raw material for concentration and subsequent recovery of titanium compounds from an iron leaching process, which is present in higher amounts, about $30 \%$ by weight. To obtain a greater understanding about the leaching kinetics, the information of the kinetic data of this process is very important. In this context, the main objective of this work is the development of a mathematical model that is able to fit the experimental data (conversion / extraction iron, titanium and aluminum) of the leaching process by which is possible to obtain the main kinetic parameters such as the activation energy and the velocity of chemical reactions as well as the controlling step of the process. The development of the mathematical model was based on the model of core decreasing. The obtained model system of ordinary differential equations was able to fit the experimental data obtained from the leaching process, enabling the determination of the controlling step, the rate constants and the activation energies of the leaching process.

Keywords: red mud, kinetics parameters, leaching

\section{NOMENCLATURE}

$a_{p} \quad$ Is the area of a particle of red mud, $m$

b Is the coefficient stoichiometric

d Is the coefficient stoichiometric

$\mathrm{C}_{\mathrm{A}}$ Is the concentration of $\mathrm{A}, \mathrm{mol} / \mathrm{liter}$

$\mathrm{D}_{\text {ef }} \quad$ Effective diffusion coefficient, $\mathrm{m}^{2} \cdot \mathrm{s}^{-1}$

$\mathrm{K}_{\mathrm{m}} \quad$ Coefficient of mass transfer, $\mathrm{m} \cdot \mathrm{s}^{-1}$

$N_{\mathrm{A}}$ The flow of the reagent in the liquid film, $\mathrm{mol} /\left(\mathrm{m}^{2} . \mathrm{s}\right)$

$N_{\text {B0 }} \quad$ The initial moles of iron

$N_{\mathrm{C} 0} \quad$ The initial moles of titanium

$N_{\text {D0 }} \quad$ The initial moles of aluminum

$\mathrm{q}_{\mathrm{A}} \quad$ The reaction rate, $\mathrm{mol} / \mathrm{s}$

$\mathrm{R} \quad$ Radius of a sphere, $\mathrm{m}$

$\mathrm{R}_{\mathrm{T}} \quad$ The total resistance based on the resistance of the sum in the liquid film, the diffusion process

resistance and resistance to chemical reaction

rc $\quad$ Core radius unreacted, $\mathrm{m}$.

$\mathrm{r}_{\mathrm{B}} \quad$ Particle radius $\mathrm{B}\left(\mathrm{Fe}_{2} \mathrm{O}_{3}\right), \mathrm{m}$

$\mathrm{r}_{\mathrm{C}} \quad$ Particle radius $\mathrm{C}\left(\mathrm{TiO}_{2}\right), \mathrm{m}$

$\mathrm{r}_{\mathrm{D}} \quad$ Particle radius $\mathrm{D}\left(\mathrm{Al}_{2} \mathrm{O}_{3}\right), \mathrm{m}$

$r_{p} \quad$ Particle radius, $m$

$\mathrm{Si} \quad$ Sum of areas, $\mathrm{m}^{2}$

$\mathrm{C}_{\mathrm{A} \infty}$ Is the concentration of A away from of the liquid film, $\mathrm{mol} / \mathrm{L}$.

$C_{A_{S}}$ Is the concentration of $\mathrm{A}$ on the particle surface, $\mathrm{mol} / \mathrm{L}$.

$\mathrm{S}_{\mathrm{B}} \quad$ Surface area of B, $\mathrm{m}^{2}$

$\mathrm{S}_{\mathrm{C}} \quad$ Surface area of $\mathrm{C}, \mathrm{m}^{2}$
$\mathrm{S}_{\mathrm{D}} \quad$ Surface area of $\mathrm{D}, \mathrm{m}^{2}$

$\mathrm{V}_{\mathrm{B}} \quad$ The volume $\mathrm{B}, \mathrm{m}^{3}$

$\tau$ The time required to complete reaction of a particle, s.

$\mathrm{X}_{\mathrm{B}} \quad$ Fractional conversion of reactant $\mathrm{B}, \%$.

$\mathrm{X}_{\mathrm{C}} \quad$ Fractional conversion of reactant $\mathrm{C}, \%$.

$\mathrm{X}_{\mathrm{D}} \quad$ Fractional conversion of reactant $\mathrm{D}, \%$.

E Activation energy, $\mathrm{KJ} / \mathrm{mol}$

\section{Greek symbols}

$\alpha$ relationship between the numbers of initial moles of iron, titanium and aluminum to the volume of the reaction

$\begin{array}{ll}\rho_{\text {B }} & \text { Density of B, } \mathrm{mol} / \mathrm{m}^{3} \\ \rho_{\mathrm{C}} & \text { Density of C, } \mathrm{mol} / \mathrm{m}^{3} \\ \rho_{\mathrm{C}} & \text { Density of } \mathrm{D}, \mathrm{mol} / \mathrm{m}^{3}\end{array}$

\section{Subscripts}

S The particle surface

p Surface of the core unreacted

$\infty \quad$ Distant location of the liquid film

\section{INTRODUCTION}

The industrial unit Alumina do Norte do Brazil S/A (Hydro- ALUNORTE), located in the state of Pará, created to produce and market alumina, aluminum oxide, $\mathrm{Al} 2 \mathrm{O} 3$, together with miner Rio do Norte S/A (MRN) aluminum Brasileiro S/A 
(ALBRAS) and Hydroelectric Tucuruí complete cycle of aluminum production in the state, and although the production of aluminum has a great socio-economic importance to the state, it generates a large amount of solid residue called red mud (LV), thereby causing environmental impacts. According to Magalhães (2012), some studies indicate that the red mud can be used as a source of raw material for the concentration and recovery of valuable metals such as titanium, thereby making it a raw material for the production thereof.

One theory for the uncatalyzed reactions of particles surrounded by a fluid which react with may be treated considering two ideal models simple: progressive conversion model and model without reaction in the core (Balarini, 2009). For the progressive conversion model assumes that reactant fluid enters and reacts in the particle at all times, most likely with different velocities and in different places. Thus, the solid reactant is converted continuously and progressively within the particle. In the model without reaction in the core is assumed that the reaction occurs primarily on the outer surface of the particle. The reaction zone then moves toward the center of the solid and may leave material for the behind completely converted and inert solid (ash), so that at any instant, there is a core of unreacted material which decrease in size during the reaction. However, the outline of this unreacted core does not always have a definite form but, nevertheless, there is evidence that, in most cases, the model without reaction in the core is closer of real particles than progressive conversion model (Levenspiel, 1999).

In this context, based on the unreacted core model, was performed a kinetic study of reaction solid-liquid (leaching) between the red mud and sulfuric acid solutions in order to extract iron, titanium concentration aiming in the red mud, and to a greater understanding of how to process the extraction of iron oxide, was carried out mathematical modeling of the extraction process, in order of obtain a model which would fit the experimental data, and capable of allowing the obtention of kinetic parameters such as: activation energy, rate constant for the reaction, well how the controller stage of the extraction process.

\section{MATHEMATICAL MODELING OF LEACHING PROCESS RED MUD}

According to the unreacted core model the stages main of a process of extracting metals from a mineral matrix can be described as follows: Stage 1Diffusion of the reactant fluid A through the layer surrounding the particle to the surface of the solid. Stage 2 - penetration and diffusion of A through the ash layer to the surface of the unreacted core. Stage 3 - Reaction of the fluid A with the solid in the reaction surface. Stage 4 - Diffusion of the fluid B product through the ashes back to the outer surface of the solid. Stage 5 - Diffusion of B through of the layer of fluid back for into the solution. Main Assumptions of the mathematical model, are:

-The mathematical modeling is performed considering that the red mud particles are all spherical;

- Considering the leaching process as a reaction system solid-fluid, the mathematical model is developed based on the unreacted core model, then the mathematical modeling is performed using the following stages: Flow of reactant $\mathrm{A}$ in the liquid film; Flow of the reactant $A$ in the region $r_{c}<r<R$; Reaction of the reagent $A$ on the surface of $r_{c}$, where $r_{c}$ is the radius of the core of the unreacted particle and $\mathrm{R}$ is the radius of the particle;

-It is considered that only the iron oxide is being extracted from red mud;

-The variables main affecting the extraction process are temperature and concentration of sulfuric acid;

-The extraction process is governed by the third stage, ie, the reaction of the fluid $\mathrm{A}\left(\mathrm{H}_{2} \mathrm{SO}_{4}\right)$ with red mud particles in the reaction surface.

\section{Development Of The Mathematical Model}

The flow of reagent $\mathrm{A}$ in the liquid film may be given under the definition of a mass transfer coefficient (similar to Newton's law of cooling). For the case in question the mass flow of A to the surface is given by:

$$
N_{A_{\infty-P}}=K_{m}\left(C_{A \infty}-C_{A_{S}}\right)
$$

where $N_{A_{\infty-P}}$ is the flow of the reagent away from of the liquid film, $\mathrm{mol} /\left(\mathrm{m}^{2} . \mathrm{s}\right), \mathrm{K}_{\mathrm{m}}$ is the coefficient of mass transfer in the liquid film, $m . s^{-1}, C_{A \infty}$ is the concentration of A away from of the liquid film, $\mathrm{mol} / \mathrm{L}$, and $C_{A_{s}}$ is the concentration of $\mathrm{A}$ on the particle surface. mol/L. For the case in question, ie, particles red mud were all considered with spherical shape, and as the flow is variable for the spherical geometries, we will opt to work, for all cases, derived from Eq. (1) in terms of rate, thus, Eq.(1), becomes:

$$
q_{\mathrm{A}_{\infty-p}}=a_{p} \times N_{\mathrm{A}_{\infty-p}}=4 \pi R^{2} K_{m}\left(C_{A \infty}-C_{A S}\right)
$$

where $q_{\mathrm{A}_{\mathrm{o}-\mathrm{P}}}$ is the taxa of reaction away from of the liquid film, mol/s, $\mathrm{R}$ is the radius of a sphere, $\mathrm{m}$, and $a_{p}$ is the area of a particle of red mud. $\mathrm{m}^{2}$.

The rate on the region $r_{c}<r<R$ can be obtained according to the Fick's law of diffusion:

$$
q_{A_{p}}=\frac{4 \pi D_{e f}}{\left(\frac{1}{r_{c}}-\frac{1}{R}\right)} \times\left(C_{A_{s}}-C_{A_{C}}\right)
$$


where $q_{A_{p}}$ is the surface reaction rate, $D_{e f}$ is the diffusion coefficient, $\mathrm{m}^{2} . \mathrm{s}^{-1}, r_{c}$ is the core radius unreacted, $\mathrm{m}$, and $C_{\mathrm{A}_{C}}$ is the concentration of the acid on the surface.

The reactions which occur on the surface $r_{c}$ are the reaction between iron $\left(\mathrm{Fe}_{2} \mathrm{O}_{3}\right)$ and sulfuric acid solution, well as, may happen the acid reaction with titanium $\left(\mathrm{TiO}_{2}\right)$ and aluminum $\left(\mathrm{Al}_{2} \mathrm{O}_{3}\right)$. The total rate of consumption of the reagent $A$ at the surface $r_{C}$ can be written as follows:

$-\frac{1}{S i} \frac{d N_{A_{\text {TOTAL }}}}{d t}=R_{A_{\text {TOTAL }}}$

$=K_{1} \frac{S_{B}}{S i} C_{A}\left|r_{B}+K_{2} \frac{S_{C}}{S i} C_{A}\right| r_{C}+K_{3} \frac{S_{D}}{S i} C_{A} \mid r_{D}$

where $\mathrm{K}_{1}, \mathrm{~K}_{2}$ and $\mathrm{K}_{3}$ are the kinetic constants of iron, titanium and aluminum, respectively, $\mathrm{m} / \mathrm{s}$, $S_{B}, S_{C}$ and $S_{D}$ are the surfaces area $\mathrm{B}, \mathrm{C}$ and $\mathrm{D}$, respectively, $\mathrm{m}^{2}, C_{A}\left|r_{B} \approx C_{A}\right| r_{C} \approx C_{A}\left|r_{D} \approx C_{A}\right| \bar{r}$ are the concentrations of $\mathrm{A}$ of the unreacted core and $\mathrm{Si}$ is the total area, $\mathrm{m}^{2}$.

Consequently the Equation of the rate for the consumer in the $\mathrm{r}_{\mathrm{c}}$ surface, takes the following form:

$$
q_{\mathrm{A}_{\text {TOTAL }}}=4 \pi \bar{r}^{2}\left(\mathrm{~K}_{1} \xi_{\mathrm{B}}+\mathrm{K}_{2} \xi_{C}+\mathrm{K}_{3} \xi_{D}\right) \mathrm{C}_{\mathrm{A} \bar{r}}(5)
$$

where $\xi_{B}=\left(\frac{r_{B}}{\bar{r}}\right)^{2} ; \xi_{C}=\left(\frac{r_{C}}{\bar{r}}\right)^{2} ; \xi_{D}=\left(\frac{r_{D}}{\bar{r}}\right)^{2}$

Making an analogy electrical with mass transfer process with chemical reaction (processes in series), we can find, derived from Eq. (5), the total resistance, $\mathbb{R}_{T}^{*}$, based on the liquid film in the process of diffusion and chemical reaction, is written as:

$\mathbb{R}_{T}^{*}=\frac{1}{k_{m}}+\frac{\left(1-\left(\frac{\bar{r}}{r_{P}}\right)\right) r_{P}}{\left(\frac{\bar{r}}{r_{P}}\right) D_{e f}}+\frac{1}{\left(\frac{\bar{r}}{r_{P}}\right)^{2}\left(\xi k_{1}+\xi k_{2}+\xi k_{3}\right)}$

where $\bar{r}=\frac{\left(\mathrm{r}_{\mathrm{B}}+\mathrm{r}_{\mathrm{C}}+r_{D}\right)}{3}$ and $r_{P}$ is the radius of particle. In this case, the rate, $q_{A}$, can be given by the equation below:

$$
q_{A_{\text {TOTAL }}}=\frac{C_{A \infty}}{\mathbb{R}_{T}}=U_{A} C_{A \infty}
$$

where $U_{A}=\frac{4 \pi r_{p}^{2}}{\mathbb{R}_{T}^{*}}$. The substituting Eq. (7) into

Eq. (5) is written as:

$$
C_{A_{\bar{T}}} \approx \frac{C_{A \infty}}{\left(\bar{r} / r_{P}\right)^{2} \mathbb{R}_{T}^{*}\left(\mathrm{~K}_{1} \xi_{B}+\mathrm{K}_{2} \xi_{C}+\mathrm{K}_{3} \xi_{D}\right)}
$$

According Balarini (2009), the chemical stage is usually more sensitive to temperature than the physical stage, therefore, experiments at different temperatures can easily distinguish between gray or diffusion layer on one side, and chemical reaction on the other hand, as driver stage. In this case, $\mathbb{R}_{T}^{*}$ will be represented by the last term of the Eq. (6).

$$
\mathbb{R}_{T}^{*}=\frac{1}{\left(\bar{r} / r_{P}\right)^{2}\left(\mathrm{~K}_{1} \xi_{B}+\mathrm{K}_{2} \xi_{C}+\mathrm{K}_{3} \xi_{D}\right)}
$$

It is known that $C_{A \infty}$ in the Eq. (8) varies with time, Bayrak (2005) in his study of natural magnesite dissolution kinetics of gluconic acid solutions affirms that this concentration can be represented as follows:

$$
C_{i}=C_{i_{0}}\left(1-\sum_{i} \frac{N_{i 0}}{V b} X_{i}\right)
$$

Based on Balarini (2009) and adapting to the case in question, the Eq. (10) takes the following form:

$$
\mathrm{C}_{\mathrm{A} \overline{\mathrm{r}}}=C_{\mathrm{A} \infty} \approx C_{\mathrm{A}(0)}\left(1-\alpha_{0} X_{B}-\alpha_{1} X_{C}-\alpha_{2} X_{D}\right)
$$

where, $\alpha_{0}=N_{B_{0}} /\left(V C_{A_{0}} b\right) ; \alpha_{1}=N_{C_{0}} /\left(V C_{A_{0}}\right)$ and $X_{i}$ is the fraction of a particular component, $X_{B}$, $X_{C}$ and $X_{D}, \%, N_{B_{0}}$ the initial moles of iron, $N_{C_{0}}$ the initial moles of aluminum, b the stoichiometric coefficient of iron and $V$ the reaction volume, $\mathrm{m}^{3}$.

The mathematical models are usually given in terms of extraction of the metals of interest (conversion), in this case, the reaction rate for the components B, C and D are represented as follow:

$$
\begin{aligned}
& \frac{d x_{B}}{d t}=\tilde{\mathbb{C}}_{1}\left(1-x_{B}\right)^{\frac{2}{3}}\left(1-\alpha_{0} x_{B}-\alpha_{1} x_{C}-\alpha_{2} x_{D}\right) \\
& \frac{d x_{C}}{d t}=\tilde{\mathbb{C}}_{2}\left(1-x_{C}\right)^{\frac{2}{3}}\left(1-\alpha_{0} x_{B}-\alpha_{1} x_{C}-\alpha_{2} x_{D}\right)
\end{aligned}
$$




$$
\begin{gathered}
\frac{d x_{D}}{d t}=\tilde{\mathbb{C}}_{3}\left(1-x_{D}\right)^{\frac{2}{3}}\left(1-\alpha_{0} x_{B}-\alpha_{1} x_{C}-\alpha_{2} x_{D}\right) \\
t=0 \Rightarrow x_{B}=x_{C}=x_{D}=0
\end{gathered}
$$

where $\mathbb{C}_{1}=\frac{3 b C A \infty(0) K_{1}}{\rho_{B} r_{p}} ; \tilde{\mathbb{C}}_{2}=\frac{3 d C A \infty(0) K_{2}}{\rho_{c} r_{p}}$

and $\tilde{\mathbb{C}}_{3}=\frac{3 b C A \infty(0) K_{3}}{\rho_{D} r_{p}}$

where $\rho_{B}, \rho_{C}$ and $\rho_{D}$ are molar density of iron, titanium and aluminum, respectively, $\mathrm{mol} / \mathrm{m}^{3}$.

To solve the mathematical model represented by Eqs. (12), (13) and (14) was built a code in FORTRAN language in 2003, where we used the IMSL routine IVPAG that is appropriate for solving of ordinary systems ordinary differential equations with nature " stiff". This code was run on a Pentium Intel (R) Core (TM) Quad 2.66 GHz platform. Also for the solution of the system of values $K_{1}, K_{2}$ and $\mathrm{K}_{3}$ were estimated, to subsequently obtain $\tilde{\mathbb{C}}_{1}, \tilde{\mathbb{C}}_{2}$ and $\tilde{\mathbb{C}}_{3}$ and consequently values of the $X_{B}(t), X_{C}(t)$ and $X_{D}(t)$ were obtained.

After this process the estimated, theoretical values of $\mathrm{K}_{1}, \mathrm{~K}_{2}$ and $\mathrm{K}_{3}$ were fitted with the experimental data for later obtaining the Arrhenius parameters .We used experimental data obtained from the leaching process at $900^{\circ} \mathrm{C}$ calcined red mud order to extraction and / or concentration of titanium present in the waste to feed the developed mathematical model. The leaching process was conducted at the temperatures 60,80 and $90^{\circ} \mathrm{C}$ using as a leaching agent in the sulfuric acid concentrations of 20 and $30 \%$.

\section{RESULTS AND DISCUSSION}

Effect of the temperature and concentration in the reaction constant $K_{1}$ and $K_{2}$

In the Table 1 can be observed values of the speed constant $\mathrm{K}_{1}$ of reaction of the red mud calcined at $900{ }^{\circ} \mathrm{C}$ (LV900). In this Table 1, it can be observed (for both acid concentrations studied) which reaction constant increases with temperature, as expected.

Table 1. Behavior of $\mathrm{K}_{1}$ with the concentration and temperature of leaching (LV-900).

\begin{tabular}{|c|c|c|}
\hline Temperature $(\mathrm{K})$ & $\mathrm{K}_{1} 20 \%(\mathrm{~m} / \mathrm{s})$ & $\mathrm{K}_{1} 30 \%(\mathrm{~m} / \mathrm{s})$ \\
\hline 333.16 & $1.76 \mathrm{E}-10$ & $1.23 \mathrm{E}-09$ \\
\hline 353.16 & $9.48 \mathrm{E}-09$ & $6.32 \mathrm{E}-09$ \\
\hline 363.16 & $2.07 \mathrm{E}-08$ & $9.48 \mathrm{E}-09$ \\
\hline
\end{tabular}

The behavior of the reaction constant $K_{1}$ with acid concentration can be analyzed as follows:

i) For $\mathrm{T}=333.16 \mathrm{~K}, \mathrm{~K}_{1}$ decreases with concentration;

ii) For $\mathrm{T}=353.16 \mathrm{~K}, \mathrm{~K}_{1}$ decreases with concentration;

iii) For $\mathrm{T}=363.16 \mathrm{~K}, \mathrm{~K}_{1}$ decreases with concentration.

In this case the red mud calcined at $900{ }^{\circ} \mathrm{C}$ sodium participated of the reactions of calcination and formed a glassy phase. Thus, it is suggested that the mechanism of reaction suffer interference of the following factors that reduce the rate of reaction with increasing concentrations of acid, such factors are: a) the common ion effect, and b) the ionic dissociation of sulfuric acid which is reduced drastically with increasing concentration, (VOGEL, 1989).

Table 2 shows the results speed constant $K_{2}$ of reaction, which has similar behavior to $K_{1}$.

Table 2. Behavior of $K_{1}$ with the concentration and temperature of leaching (LV-900).

\begin{tabular}{|c|c|c|}
\hline Temperature $(\mathrm{K})$ & $\mathrm{K}_{2} 20 \%(\mathrm{~m} / \mathrm{s})$ & $\mathrm{K}_{2} 30 \%(\mathrm{~m} / \mathrm{s})$ \\
\hline 333.16 & $4.32 \mathrm{E}-10$ & $2.28 \mathrm{E}-10$ \\
\hline 353.16 & $7.20 \mathrm{E}-10$ & $4.92 \mathrm{E}-10$ \\
\hline 363.16 & $8.88 \mathrm{E}-10$ & $6.84 \mathrm{E}-10$ \\
\hline
\end{tabular}

In the Figures (1) and (2) for the iron and titanium are shown respectively in curves $\operatorname{lnK}_{1}$ and $\ln K_{2}$ versus $1 / T$ based on the Arrhenius equation for the red mud in the two acid concentrations studied.

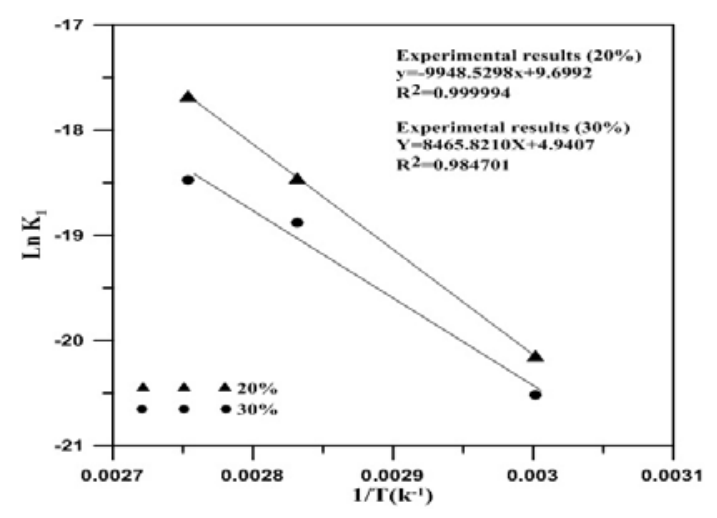

Figure 1. Obtaining the kinetics parameters of the Arrhenius equation of the iron oxide.

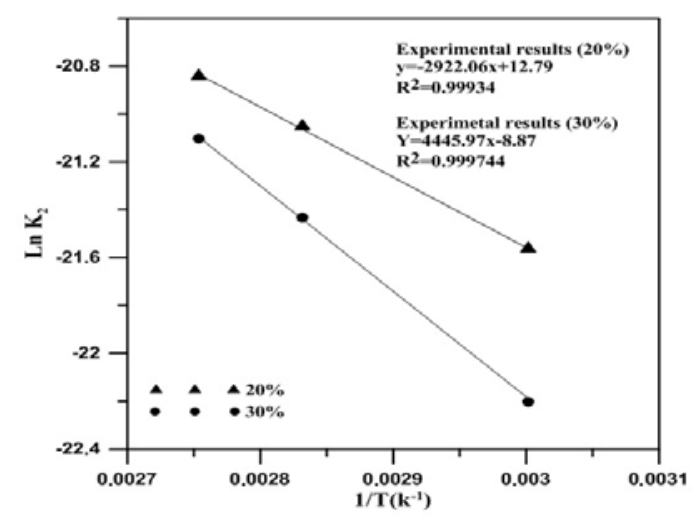

Figure 2. Obtaining the kinetics parameters of the Arrhenius equation of the titanium oxide. 
From Figures 1 and 2 it was possible to obtain the kinetic parameters of activation energy and frequency factor.

Table 3 shows the values of parameters kinétics the Arrhenius equation for the iron obtained from Figure 1 . The value of activation energy shows that for the reaction with iron oxide leaching kinetics is controlled by the chemical reaction.

Table 3. Kinetics parameters of the Arrhenius equation for iron .

\begin{tabular}{|c|c|c|}
\hline$\%$ de $\mathrm{H}_{2} \mathrm{SO}_{4}$ & $\mathrm{E}(\mathrm{KJ} / \mathrm{mol})$ & $\mathrm{K}_{0}(\mathrm{~m} / \mathrm{s})$ \\
\hline 20 & 82.71 & 16305.2 \\
\hline 30 & 70.38 & 139.06 \\
\hline
\end{tabular}

Table 4 it is noted that for the reaction with the titanium oxide the activation energy values are below of $25 \mathrm{KJ} / \mathrm{mol}$ for concentration of $20 \%$ sulfuric acid, suggests that the mass transport is the controlling step in the process.

Table 4. Kinetics parameters of the Arrhenius equation for titanium.

\begin{tabular}{|c|c|c|}
\hline$\% \mathrm{de}_{2} \mathrm{SO}_{4}$ & $\mathrm{E}(\mathrm{KJ} / \mathrm{mol})$ & $\mathrm{K}_{0}(\mathrm{~m} / \mathrm{s})$ \\
\hline 20 & 24.29 & $2.79 \mathrm{E}-06$ \\
\hline 30 & 36.96 & $14.29 \mathrm{E}-05$ \\
\hline
\end{tabular}

\section{CONCLUSIONS}

In this work, was developed a mathematical model based on total resistance to mass transfer in the leaching process. This model has been constructed taking into consideration the resistance at the liquid film, the resistance in the region of ash and resistance due to chemical reaction at the surface of the unreacted core.

An analytical solution for the model was determined considering that iron is prevalent in the extraction process and that the reaction rate controls the leaching process. The model then was fit to experimental data in order to determine the kinetic constants and the parameters of the Arrhenius law for each concentration studied.

For the case of red mud calcined at $900{ }^{\circ} \mathrm{C}$ the sodium was consumed, and thus, it is suggested that the reaction mechanism only suffer interference of the common ion effect and ionic dissociation of sulfuric acid.

The results of activation energy for the reaction with the iron exceeds $40 \mathrm{KJ} / \mathrm{mol}$, which according to the literature, the values above this shows that the chemical reaction is the step of controlling the process. For reactions with titanium oxide was observed that the values for the activation energy is less than $25 \mathrm{KJ} / \mathrm{mol}$ for the $20 \%$ concentration sulfuric acid, according to propose Prosser (1996), mass transport is step control process of leaching.
Balarini, J. C., 2009, Kinetic Study Ustulado of Votorantim Metais Zinco: Catalytic Mechanism not Associated with the Characterization and Determination of Residence Times, Doctoral Thesis, School of Engineering, Minas Gerais, MG, Brazil.

Bayrak, B., 2005, Investigation of Dissolution Kinetics Natural Magnesite in Gluconic Acid Solutions, Journal of Chemical Engineering, Vol. 117, No. 1, pp. 109-115.

Bhatnagar, S, S., and Sundara Rao, A. L., 1945, Pilot Plant for the Recovery of Titanium Dioxide from Bauxite Sludge, Journal of Scientific and Industrial Research, Vol. 4, pp. 378-381.

Levenspiel, O., 2000, Engineering of Chemical reactions, Edgar Blucher Book.

Magalhães, E, M, 2012, Estudo da Extração de Compostos de Ferro da Lama Vermelha Visando a Extração e/ou Recuperação de Compostos de Titânio, Doctoral Thesis, PRODERNA/ITEC, Belém, PA. (in Portuguese)

Kasliwal, P, and Sai, P. S. T., 1999, Enrichment of Titanium Dioxide in Red Mud: a Kinetic Study, Hydrometallurgy, Vol. 53, pp. 73-87.

Vogel, A, I., 1989, Quantitative Chemical Analysis, Textbook.

\section{REFERENCES}

